\title{
Analysis of sewage sludge using an experimental prompt gamma neutron activation analysis (pgnaa) set-up with an am-be source
}

\author{
Z. Idiri \\ Centre de Recherche Nucleaire d'Alger, 02 Bd Frantz Fanon, B.P.399, Alger-Gare, Algeria \\ idiiridz@yahoo.fr \\ F. Redjem \\ Universite des Sciences et de la Technologie Houari Boumediene, Bab Ezzouar, Alger, Algeria \\ f-redjem@yahoo.fr \\ N. Beloudah \\ Universite des Sciences et de la Technologie Houari Boumediene, Bab Ezzouar, Alger, Algeria \\ b_belouadah@yahoo.fr
}

Published 1 September 2016

\begin{abstract}
An experimental PGNAA set-up using a $1 \mathrm{Ci} \mathrm{Am}-\mathrm{Be}$ source has been developed and used for analysis of bulk sewage sludge samples issued from a wastewater treatment plant situated in an industrial area of Algiers. The sample dimensions were optimized using thermal neutron flux calculations carried out with the MCNP5 Monte Carlo Code. A methodology is then proposed to perform quantitative analysis using the absolute method. For this, average thermal neutron flux inside the sludge samples is deduced using average thermal neutron flux in reference water samples and thermal flux measurements with the aid of a ${ }^{3} \mathrm{He}$ neutron detector. The average absolute gamma detection efficiency is determined using the prompt gammas emitted by chlorine dissolved in a water sample. The gamma detection efficiency is normalized for sludge samples using gamma attenuation factors calculated with the MCNP5 code for water and sludge. Wet and dehydrated sludge samples were analyzed. Nutritive elements (Ca, N, P, K) and heavy metals elements like $\mathrm{Cr}$ and $\mathrm{Mn}$ were determined. For some elements, the PGNAA values were compared to those obtained using Atomic Absorption Spectroscopy (AAS) and Inductively Coupled Plasma (ICP) methods. Good agreement is observed between the different values. Heavy element concentrations are very high compared to normal values; this is related to the fact that the wastewater treatment plant is treating not only domestic but also industrial wastewater that is probably rejected by industries without removal of pollutant elements. The detection limits for almost all elements of interest are sufficiently low for the method to be well suited for such analysis.
\end{abstract}

Keywords: Sewage sludge; industrial wastewater; PGNAA.

\section{Introduction}

Prompt Gamma Neutron Activation Analysis (PGNAA) with isotopic neutron sources is a very useful technique for in-situ and on-line analysis in different fields. ${ }^{1-3}$ This is made possible not only by easily transportable isotopic neutron sources but also with the

This is an Open Access article published by World Scientific Publishing Company. It is distributed under the terms of the Creative Commons Attribution 3.0 (CC-BY) License. Further distribution of this work is permitted, provided the original work is properly cited. 
development of very efficient portable gamma ray detectors and associated electronics. In a previous work, ${ }^{4}$ a prompt gamma neutron activation analysis set-up for water reject analysis has been described and optimized using Monte Carlo neutron transport calculations with the MCNP5 code. ${ }^{5}$ The optimization concerned the dimensions of the Perspex sample cylindrical container. In this set-up, the $1 \mathrm{Ci} \mathrm{Am}-\mathrm{Be}$ neutron source emitting around $2.2 \times 10^{6} \mathrm{n} / \mathrm{s}$ is confined in a Perspex tube immersed in the center of the sample. The maximum average thermal neutron flux was looked for in the sample with the constraints that neutron damage, neutron and gamma rays doses, and pulse pile-up must be at reasonable levels.

The optimized set-up has been achieved and is shown in Fig. 1. The optimal sample dimensions are $40 \mathrm{~cm}$ for the diameter and $20 \mathrm{~cm}$ for the height. To detect the prompt emitted gamma rays, a high purity germanium (HPGe) detector is placed at the surface of the Perspex sample container. A Perspex tube containing the Am-Be source is immersed in the sample with its end situated exactly at the center of the sample. The set-up has been characterized and then applied to liquid samples analysis, especially domestic wastewater and industrial phosphoric acid. ${ }^{6}$

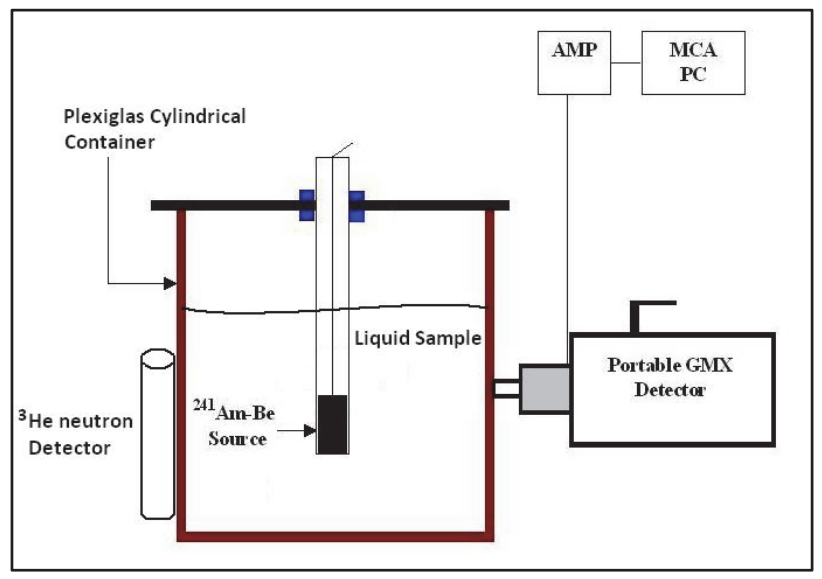

Fig. 1. Experimental PGNAA set-up developed at the Nuclear Research Center of Algiers.

Presently, there is a growing concern over the environmental pollution problems that should be managed carefully according to the existing relevant national laws. In Algeria, a new regulation has recently been released ${ }^{7}$ to regulate and control the environmental pollutions discharged into water issued from sewage and industrial pollutants such as factories, mills, refineries, etc. Generally, wastewaters contain aqueous domestic wastes as well as surface drainage waters and, in many cases, a component of treated and untreated industrial effluents. A wide range of substances are concentrated in wastewaters, such as metals, poorly biodegradable trace organic compounds and potentially pathogenic organisms. If not managed properly, untreated discharged wastewater effluents constitute a high risk to environmental resources and human health. To tackle such typical problems, PGNAA with an isotopic neutron source can be 
considered as a suitable nuclear technique for in situ, rapid and continuous survey of such pollutants.

Sewage treatment, or domestic wastewater treatment, is the process of removing contaminants from wastewater and household sewage, both effluents and domestic. It includes physical, chemical, and biological processes to remove physical, chemical, and biological contaminants. Its objective is to produce an environmentally-safe fluid waste stream (or treated effluent) and a solid waste (or treated sludge) suitable for disposal or reuse (usually as farm fertilizer).

In this work, we have used the above cited PGNAA experimental set-up to analyze hydrated and dehydrated sewage sludge provided by a wastewater treatment plant in Algiers. For quantitative analysis, the absolute method was used. Some nutritive elements $(\mathrm{Ca}, \mathrm{N}, \mathrm{P}, \mathrm{K})$ and heavy metals $(\mathrm{Cr}, \mathrm{Mn})$ were determined, and the results were compared to those obtained by Inductively Coupled Plasma (ICP) and Atomic Absorption Spectroscopy (AAS) methods.

\section{Experimental}

The prompt emitted gamma rays are detected with an ORTEC high purity germanium (HPGe) detector having 15\% relative efficiency and $2 \mathrm{keV}$ resolution at $1332.5 \mathrm{keV}$ of ${ }^{60} \mathrm{Co}$. The detector is placed in the center of the sample container directly touching its external surface. In order to monitor the thermal neutron flux inside the sample, a ${ }^{3} \mathrm{He}$ neutron detector is placed on the opposite side of the sample container. The center of the neutron detector is adjacent to the plane perpendicular to the sample axis and passing by the center of the sample. The portable gamma-ray detector is connected to a portable Canberra Model inspector 2000 integrated digital signal processing electronic unit in which the signal coming from the preamplifier of the detector is digitized, amplified, and then processed in a multichannel analyzer. All the functions are controlled through a portable computer, and the obtained gamma ray spectra are analyzed by Canberra Genie 2000 gamma software. The inspector 2000 employs a lithium battery that allows at least 4 hours of autonomous operation. The set-up described above may be used for field analyses. The thermal neutron flux measurements were carried out with a LND $252{ }^{3} \mathrm{He}$ neutron detector of around $24 \mathrm{~cm}$ length connected to a Ludlum 16 rate meter. The yield of the neutron LND 252 neutron detector is $21.8 \mathrm{cps} / 1 \mathrm{ncm}^{-2} \cdot \mathrm{s}^{-1}$.

The gain of the linear amplifier was set so that the energy of the detected prompt gamma rays can be greater than $10 \mathrm{MeV}$. In order to correct for pulse pile-up losses, the pulser method was adopted with the pulser peak lying in the high energy part of the spectra.

\section{Sampling}

Two sludge samples have been collected: one dehydrated and one hydrated. They were packaged in plastic bags and weighed to determine their densities. The densities were found to be $1.47 \mathrm{~g} / \mathrm{cm}^{3}$ for the dehydrated sample and $0.956 \mathrm{~g} / \mathrm{cm}^{3}$ for the hydrated one. To compare the results obtained with PGNAA to those determined with chemical and 
physical analysis methods (atomic absorption and ICP), samples were freeze-dried and chemically treated to obtain solutions.

\section{Methodology}

The measured count rate $A$ of a prompt gamma ray of energy $E_{\gamma}$ emitted from a capturing nuclide in a sample of volume $\mathrm{V}$ is given by ${ }^{6}$

$$
A=\frac{1}{V} \frac{m}{M} N_{A} \theta \int_{V} d^{3} r \varepsilon\left(r, E_{\gamma}\right) \int_{0}^{\infty} P\left(E_{\gamma}, v\right) n(r, v) \sigma(v) v d v,
$$

where $\mathrm{m}$ is the element mass, $\mathrm{M}$ is the atomic mass, $\mathrm{N}_{\mathrm{A}}$ is Avogadro's number, $\theta$ is the isotopic abundance, $\varepsilon(\mathrm{r}, \mathrm{E}-\gamma)$ is the detection efficiency for the prompt gamma ray of energy $E_{\gamma}$ emitted at the location $r, P\left(E_{\gamma}, v\right)$ is the absolute gamma ray emission probability (gammas emitted per capture) of the prompt gamma rays of energy $\mathrm{E}_{\gamma}$ emitted from the nucleus capturing a neutron of speed $\mathrm{v}, \sigma(\mathrm{v})$ is the speed-dependent neutron capture cross section for the nucleus, and $n(r, v)$ is the speed-dependent neutron density at location $r$.

For a well thermalized neutron flux, the counting rate given in Eq. (1) may be written as

$$
A=\frac{m}{M} N_{A} \theta P\left(E_{\gamma}\right) \bar{\epsilon}\left(E_{\gamma}\right) \bar{\phi}_{0} g \sigma_{0},
$$

where $\bar{\phi}_{0}$ is the conventional thermal neutron flux averaged over the whole volume sample, $\sigma_{0}$ is the capture cross section at the speed of $2200 \mathrm{~m} / \mathrm{s}, \bar{\varepsilon}(\mathrm{E} \gamma)$ is the detection efficiency for the prompt gamma of energy E $\gamma$ averaged over the whole sample volume, and $g$ is the Westcott factor for the element of interest.

The absolute method used in this work consists of deducing the mass of the element of interest directly from Eq. (2). It also necessitates the knowledge of the average thermal neutron flux $\bar{\phi}_{0}$ in the unknown sample and the average gamma detection efficiency $\bar{\epsilon}$. The average thermal flux is deduced by normalizing the average thermal neutron flux in the water sample calculated with MCNP and using the flux measurements of thermal flux carried out with the ${ }^{3} \mathrm{He}$ detector on the surface sample holder respectively for water as reference sample and unknown sample.

For the average gamma detection efficiency, we determine it first for water by measuring prompt gamma rays of chlorine and using Eq. (2). Then we define a gamma ray self-attenuation factor $F_{\text {att }}$, and the average prompt gamma ray efficiency $\bar{\varepsilon}\left(E_{\gamma}\right)$ in Eq. (2) can be replaced by the following formula:

$$
\bar{\varepsilon}\left(E_{\gamma}\right)=\bar{\epsilon}_{0}\left(E_{\gamma}\right) \cdot F_{\text {att }},
$$

Where $\bar{\epsilon}_{0}\left(E_{\gamma}\right)$ is the average absolute efficiency for the PGNAA set-up without sample, and $\mathrm{F}_{\text {att }}$ is the average attenuation factor that corresponds only to the gamma ray attenuation in the sample.

If we know the raw composition of the sample to analyze, the gamma ray attenuation factors may be calculated using MCNP5. As we need only an average value of the 
attenuation factor, we assume that the gamma emission is distributed homogeneously; we calculate the probability of the gamma rays to reach the detector volume. To determine the attenuation factor, we calculate the precedent probability with and without the sample, and it follows that

$$
\mathrm{F}_{\mathrm{att}}=\frac{\mathrm{P}_{\gamma, \mathrm{s}}}{\mathrm{P}_{\gamma, \mathrm{ws}}}
$$

Where $p_{\gamma, s}$ is the probability that a gamma ray originating from the sample reaches the detector, and $\mathrm{p}_{\gamma, \mathrm{ws}}$ is the probability that a gamma ray originating from the container (without sample) reaches the detector.

According to Eq. (3), the relationship between the averaged absolute gamma ray efficiencies for water and unknown samples is given by

$$
\bar{\epsilon}_{u}\left(E_{\gamma}\right)=\bar{\epsilon}_{w}\left(E_{\gamma}\right) \cdot\left(\frac{F_{a t t, u}}{F_{a t t, w}}\right),
$$

Where $\bar{\epsilon}_{u}$ and $\bar{\epsilon}_{w}$ are averaged absolute gamma efficiencies respectively for unknown and water samples, and $F_{a t t, u}$ and $F_{a t t, w}$ are respectively the gamma-ray attenuation factors of samples.

\section{Results and Discussion}

\subsection{Optimization of sludge sample dimensions}

The diameter of the sample Perspex container of our PGNAA set-up is $40 \mathrm{~cm}$, and we could only optimize the height of the samples. We used MCNP5 to calculate the average thermal neutron flux in the sample for different sample height values. The optimum value is that corresponding to the maximum average thermal neutron flux.

The hydrated sludge contains $91 \%$ water; the rest is organic matter. Its density is $0.965 \mathrm{~g} / \mathrm{cm}^{3}$. The results for hydrated sludge are shown in Fig. 2. We can note that the optimum height is $20 \mathrm{~cm}$, similar to the optimum value for the height of the pure water sample determined in our previous work. ${ }^{4}$ 


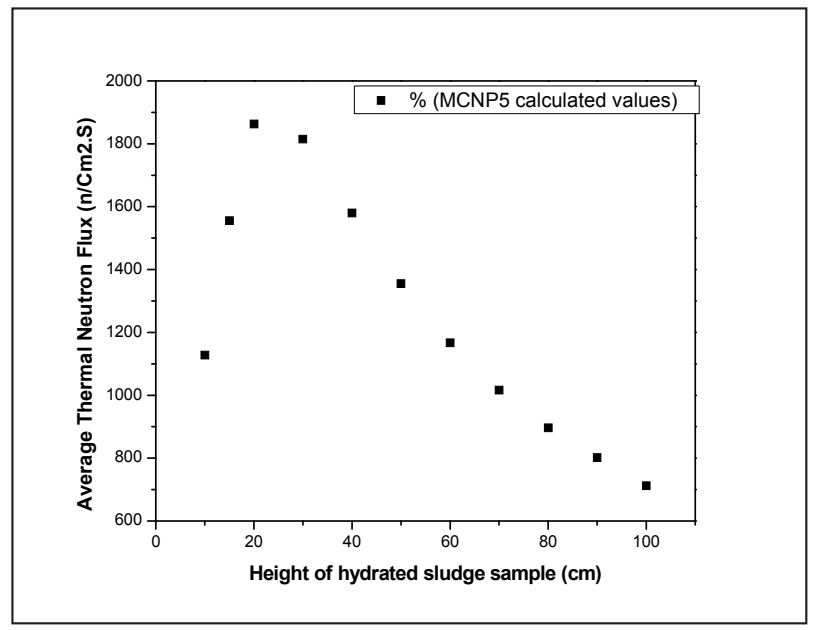

Fig. 2. Variations of average thermal neutron flux in hydrated sludge sample versus the height of the sample.

The dehydrated sludge is composed of $57 \%$ water and $43 \%$ organic matter. The variations of average thermal neutron flux in dehydrated sludge versus the height of the sample are shown in Fig. 3. As we can note, the optimum height of the sample is $40 \mathrm{~cm}$.

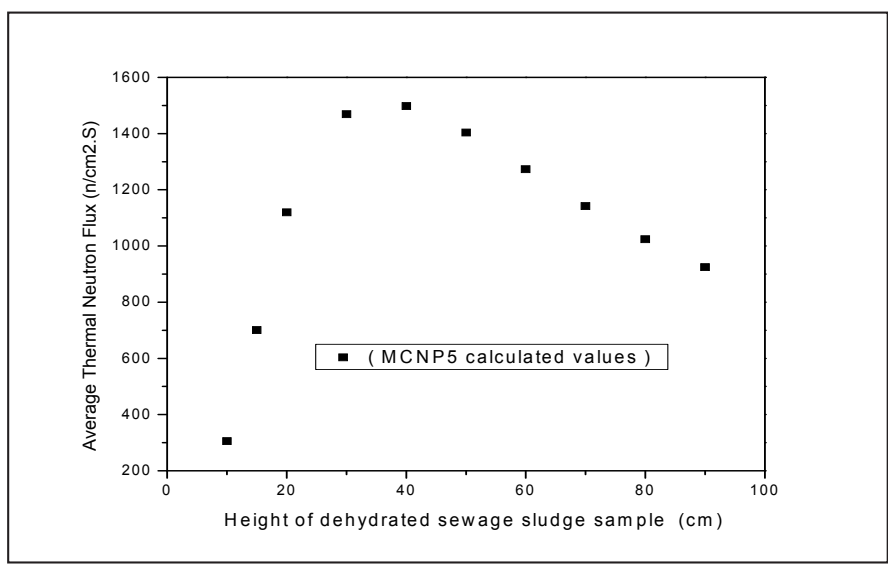

Fig. 3. Variations of average thermal neutron flux in dehydrated sludge versus the height of the sample.

\subsection{Prompt gamma neutron activation analysis of hydrated and dehydrated sewage sludge}

The average gamma detection efficiency for water reference samples is determined using prompt gamma rays of chlorine dissolved in water and Eq. (2). The average thermal neutron flux in such samples has already been calculated in our previous work using MCNP5. The average gamma detection efficiency for the $15 \%$ HPGe detector used in this work is shown in Fig. 4. 


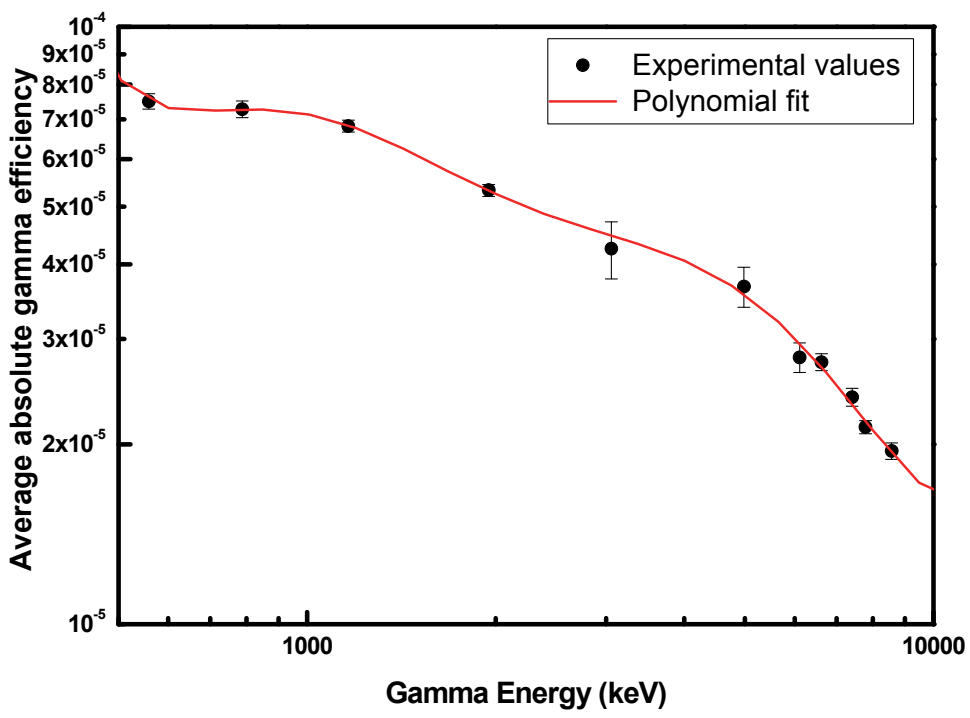

Fig. 4. Average absolute gamma detection efficiency for water samples.

The gamma attenuation factors as defined by Eq. (4) are calculated using MCNP5 for water, and for hydrated and dehydrated sludge. The results are shown in Fig. 5. As we can observe, the greater the attenuation factor, the lower the density, which is in good agreement with the definition of the factors.

The sludge samples have been irradiated and the prompt gamma spectra were collected during $14400 \mathrm{~s}$. The irradiation of the sludge samples is shown in Fig. 6. The prompt gamma spectrum of the hydrated sewage sludge is given in Fig. 7. At lower energies, the $2223 \mathrm{keV}$ gamma ray corresponding to hydrogen is very intense. This line is accompanied by two lines corresponding to single and double escape (1201 and 1712 $\mathrm{keV}$ ). At high energies, the $4430 \mathrm{keV}$ gamma ray corresponding to the gamma deexcitation of ${ }^{12} \mathrm{C}$ is the most intense. It is accompanied by two lines of single and double escape. These lines have a relatively large width at half-maximum because of the Doppler effect that characterizes the emission of de-excitation gammas of the recoil nucleus ${ }^{12} \mathrm{C}$ derived from the reaction ${ }^{9} \mathrm{Be}(\mathrm{n}, \alpha){ }^{12} \mathrm{C}$. There are also lines of $\mathrm{Fe}(6129,7631$, and $7646 \mathrm{keV}$ ) with their corresponding single and double escape lines. The calibration of the gamma detection chain was carried out using the different gamma and prompt gamma ray lines cited before. 


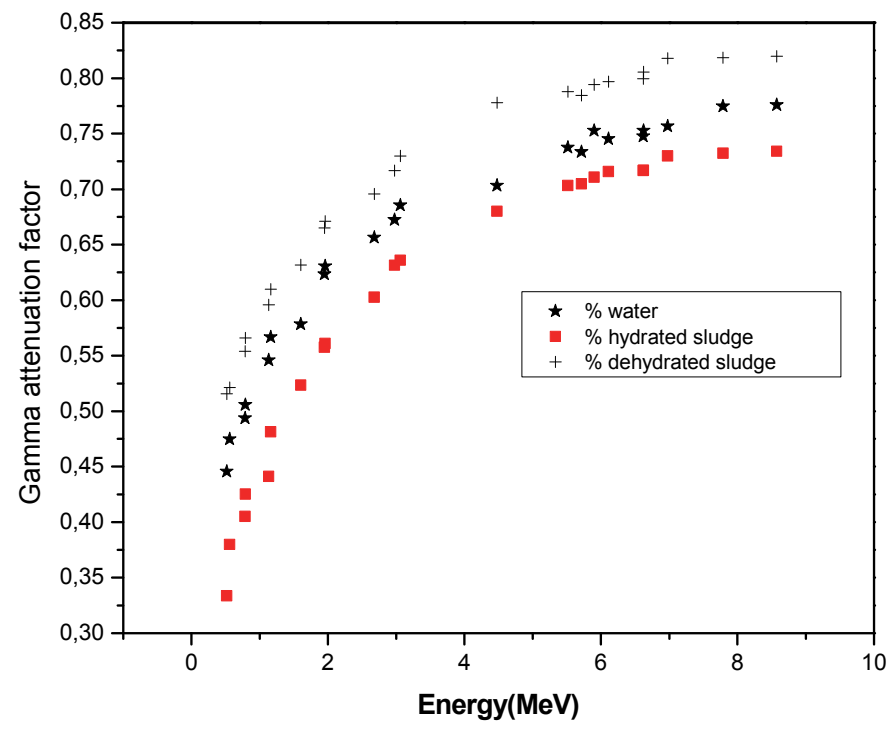

Fig. 5. Gamma attenuation factors against gamma energy for water, and hydrated and dehydrated sludge.

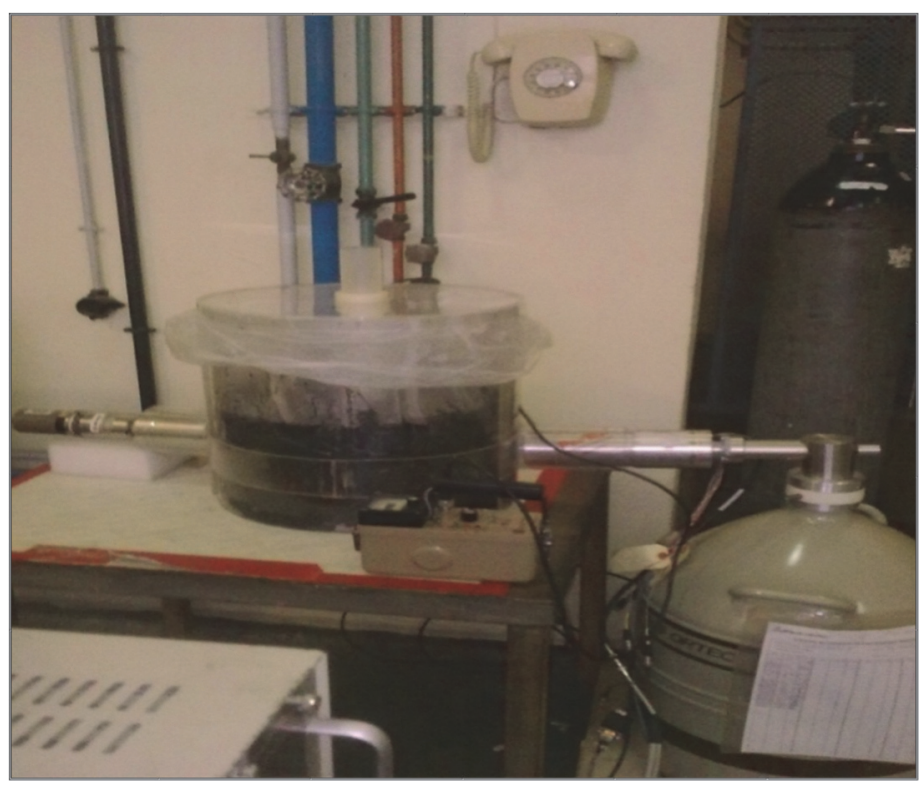

Fig. 6. Irradiation of the sewage sludge using the PGNAA set-up. 


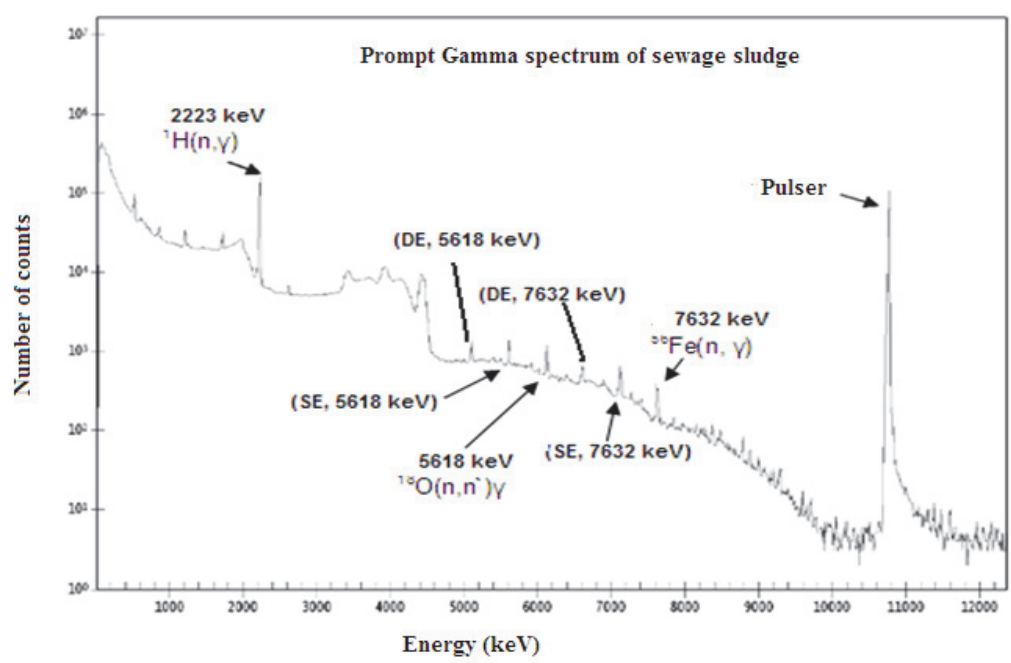

Fig. 7. Typical prompt gamma spectrum of sewage sludge.

Table 1 lists the prompt gamma-ray lines used for qualitative and quantitative analysis.

Table 1. Prompt gamma rays used for qualitative and quantitative analysis.

\begin{tabular}{ccc}
\hline Element & $\begin{array}{c}\text { Neutron capture cross section } \\
\text { (barn) }\end{array}$ & $\begin{array}{c}\text { Prompt Gammas used for identification } \\
(\mathrm{keV})\end{array}$ \\
\hline $\mathrm{Si}$ & 0.177 & 4930 \\
& & 3539 \\
$\mathrm{Cl}$ & 43.5 & 8578 \\
$\mathrm{Fe}$ & 2.25 & 7413 \\
$\mathrm{Na}$ & 0.530 & 7631 \\
$\mathrm{Cr}$ & 18.2 & 2518 \\
$\mathrm{Mn}$ & 13.36 & 8884 \\
$\mathrm{~K}$ & 2.1 & 7243 \\
& & 5684 \\
$\mathrm{~N}$ & 0.0798 & 5732 \\
& & 5297 \\
$\mathrm{P}$ & 0.172 & 10820 \\
$\mathrm{Ca}$ & 0.41 & 6785 \\
\hline
\end{tabular}

Before identifying the prompt gamma rays in sludge samples spectra, we have already determined all the prompt gamma rays of the background by analyzing the prompt gamma spectrum of the water sample and the prompt gamma spectrum originating from fast neutron interactions with all detector materials. ${ }^{7}$ The quantitative PGNAA results for hydrated and dehydrated sewage sludge are summarized in Table 2. 
Table 2. Summary of the analysis results of hydrated and dehydrated sewage sludge (Conc. = concentration, DHSS $=$ Dehydrated Sewage Sludge, HSS $=$ Hydrated Sewage Sludge, CDL $=$ Concentration Detection Limit, Norms $=$ Maximum Admitted Concentration).

\begin{tabular}{ccccccc}
\hline Element & $\sigma($ barn $)$ & $\begin{array}{c}\text { Conc. DHSS } \\
(\%)\end{array}$ & $\begin{array}{c}\text { CDL DHSS } \\
(\%)\end{array}$ & $\begin{array}{c}\text { Conc. HSS } \\
(\%)\end{array}$ & $\begin{array}{c}\text { CDL HSS } \\
(\%)\end{array}$ & $\begin{array}{c}\text { Norms } \\
(\%)\end{array}$ \\
\hline $\mathrm{Si}$ & 0.177 & $10.04 \pm 0.04$ & 0.21 & $8.03 \pm 0.05$ & 0.16 & 7.5 \\
$\mathrm{Cl}$ & 43.5 & $0.13 \pm 0.02$ & 0.02 & $0.05 \pm 0.01$ & 0.015 & 0.34 \\
$\mathrm{Fe}$ & 2.25 & $1.99 \pm 0.21$ & 0.08 & $1.34 \pm 0.24$ & 0.04 & 2.25 \\
$\mathrm{Na}$ & 0.53 & $6.7 \pm 0.6$ & 0.12 & $1.6 \pm 0.3$ & 0.02 & 0.04 \\
$\mathrm{Cr}$ & 18.2 & $1 . \pm 0.1$ & 0.1 & $0.6 \pm 0.1$ & 0.10 & 0.1 \\
$\mathrm{Mn}$ & 13.36 & $0.58 \pm 0.05$ & 0.02 & $0.3 \pm 0.02$ & 0.04 & 0.08 \\
$\mathrm{~K}$ & 2.1 & $6.8 \pm 2.6$ & 0.16 & - & - & 2.49 \\
$\mathrm{P}$ & 0.172 & $3.8 \pm 0.4$ & 0.41 & $0.35 \pm 0.13$ & 0.33 & 1.2 \\
$\mathrm{Ca}$ & 0.41 & $2.5 \pm 0.3$ & 0.1 & $2.36 \pm 0.14$ & 0.05 & - \\
$\mathrm{N}$ & 0.0075 & $12.5 \pm 1.4$ & 0.9 & - & - & 4.65 \\
\hline
\end{tabular}

We see from Table 2 that in the case of the hydrated sewage sludge, the PGNAA concentrations for the elements $\mathrm{P}, \mathrm{Fe}$, and $\mathrm{Cl}$ are less than those given by the norms (Maximum Admitted Concentrations). ${ }^{8-9}$ However, the PGNAA concentrations for Mn, $\mathrm{Cr}$, and $\mathrm{Na}$ exceed that given by the norms. This is probably due to the fact that a part of the wastewater that is treated by the plant comes from the industrial area where the wastewaters are often discharged without any treatment. In general, the concentrations found in the solid sludge are larger than those of the slurry, which is normal because the solid sludge comes from fresh (liquid) that has been dehydrated. The detection limits for elements that were measured in this work are relatively smaller compared to the concentrations usually encountered (norms), indicating that this technique is well suited to the analysis of sludge from sewage treatment.

For the dehydrated sewage sludge, chromium (Cr) was determined using the Inductively Coupled Plasma (ICP) method, while the Atomic Absorption Spectroscopy (AAS) method was used for calcium $(\mathrm{Ca})$ and iron $(\mathrm{Fe})$. The determined values were then compared to those obtained with PGNAA. The results are summarized in Table 3.

Table 3. Comparison of ICP and ASS values to PGNAA values for $\mathrm{Ca}, \mathrm{Fe}$ and $\mathrm{Cr}$ in dehydrated sewage sludge.

\begin{tabular}{cccc}
\hline Element & $\mathrm{ICP}(\%)$ & PGNAA(\%) & AAS(\%) \\
\hline $\mathrm{Fe}$ & - & $1.99 \pm 0.21$ & $1.75 \pm 0.05$ \\
& & & \\
$\mathrm{Cr}$ & $0.9 \pm 0.03$ & $1 . \pm 0.1$ & - \\
$\mathrm{Ca}$ & - & $2.5 \pm 0.3$ & $2.36 \pm 0.14$ \\
\hline
\end{tabular}

The PGNAA concentrations for $\mathrm{Cr}, \mathrm{Fe}$ and $\mathrm{Ca}$ are in good agreement with those determined using ICP and AAS methods. 


\section{Conclusion}

In this work, we have developed a method of analyzing sludge from wastewater treatment plants using PGNAA with an Am-Be source. We used an experimental set-up that consists mainly of an Am-Be source, a cylindrical sample holder and detector Plexiglas, and an HPGe gamma detector of $15 \%$ relative efficiency.

For qualitative analysis, we first identified the prompt gamma background by irradiating a sample of water. For quantitative analysis, we used the absolute method and the gamma detection efficiency determined experimentally, with the prompt gamma from chlorine dissolved in a water sample used as the reference sample. Changes in the thermal neutron flux are treated using measures of thermal neutron flux performed with a ${ }^{3} \mathrm{He}$ counter. For the gamma attenuation in the samples, we used attenuation factors calculated with the MCNP5 code. The results showed that for the dehydrated sewage sludge, the concentrations of certain elements $(\mathrm{Fe}, \mathrm{Cl})$ are lesser compared to those given by the norms, unlike those of some heavy metals $(\mathrm{Cr}, \mathrm{Mn})$ that are relatively high. High concentrations of heavy metals are probably due to the treatment of water discharges from industrial units. The concentrations found for the solid sludge are much larger than the slurry. This can be explained by the fact that the sludge solids slurries come from dehydrated sewage sludge. On the other hand, the detection limits for all elements are relatively smaller than those given in the norms, which make this technique very suitable for the analysis of wastewater treatment plant sludge.

\section{References}

1. M. Borsaru, M. Berry, M. Biggs, and A. Rojc, Nucl. Instr. Meth. B 213, 530 (2004).

2. J. Kenneth Shultis, F. Khan, B. Letellier, and Richard E. Faw, Appl. Rad. Isot. 53, 565 (2001).

3. J. Atanackovic, J. Grinyer, D.R. Chettle, S.H. Byun, Nucl. Instr. Method. B 263, 169 (2007).

4. Z. Idiri, H. Mazrou, S. Beddek, A. Amokrane, and A. Azbouche, Nucl. Instr. Meth. A 578, 279 (2007).

5. MCNP-A General Monte Carlo N-particle transport code, Version 5 (Los Alamos National Laboratory, 2003).

6. Z. Idiri, H. Mazrou, S. Beddek, and A. Amokrane, Nucl. Instr. Meth. B 268, 213 (2010).

7. G.F. Knoll, Radiation Detection and Measurement, $3^{\text {rd }}$ edn. (John Wiley and Sons, 2000).

8. Journal Officiel (J. O.), Loi 03-10 du 19 Juillet 2003, relative à la protection de l'environnement dans le cadre du développement durable, 43 (2003).

9. L. Barideau, Les Boues d'épuration, Menaces pour l'Environnement ou Matières Première pour l'Agriculture, Bull. Rech. Agro., 369 (1986). 